\title{
Adaptive backstepping control for an XY-table
}

\author{
Rob Rijs and Bram de Jager ${ }^{1}$ \\ Faculty of Mechanical Engineering \\ Eindhoven University of Technology \\ P.O. Box 513, 5600 MB Eindhoven, The Netherlands
}

\begin{abstract}
Adaptive controllers, based on backstepping, are verified on practicability, and are used to improve the control of an experimental setup: an $\mathrm{XY}$-table. Theoretical bounds for the adaptive schemes indicate strong transient performance. So far little is known about the robustness of the controllers. The robustness, with state and output feedback controllers, is verified with experiments for the XY-table.

The controllers based on state feedback showed good results. They were easy to implement and their parameter tuning appeared straightforward. The controllers based on output feedback were more difficult to implement. In experiments the range of possible tunings was restricted due to a conflict between numerical accuracy and real time requirements. Two extensions on the original control scheme are proposed. Both extensions result in more freedom in the controller design. In the allowable tuning range, the results were still inferior to the results obtained with state feedback.
\end{abstract}

Keywords: Adaptive control, backstepping, mechanical control systems.

\section{Introduction}

In the past, several robust control design methods, e.g., using adaptation, have been employed for an XY-table [1]. For all controllers used up til now the tracking error is still large due to (1) limited closed-loop bandwidth, (2) limited sampling frequency, (3) difficult to tune (adaptation) gains, and (4) model errors. Sometimes ultimate tracking is achieved only, while the transient performance may be unacceptable. A general problem of implementation of traditional adaptive control schemes is that they have hardly become tools for systematic engineering design. Each adaptive scheme leaves up to the designer the choice of various filters, design coefficients, initialization rules, and so on. It is still unclear how the adaptive system's performance, especially its transient performance, depends on these design choices.

A recently developed adaptive design method may be a solution for these shortcomings. It removes some of the disadvantages of adaptive controllers and probably some of the shortcomings of the control of the XY-table, clear guidelines for the transient performance are presented. The new method is based on recursive design procedures, referred to as backstepping [2]. Analytical bounds and simulation studies have shown that the new adaptive designs achieve better transient performance than the traditional schemes [3, Section 1.3]. With the introduction of tuning functions, Krstić et al. [4,5] got a design that removed the over-parameterization originally present.

In [3] different backstepping designs are presented: state and output feedback controllers, Lyapunov as well as estimation-based. So far, little is known about robustness of controllers based on backstepping. Therefore a question remains: What happens if some assumptions in the stability proofs are shattered, e.g., by unmodeled dynamics?

Here this question is partly answered by experiments, with controllers based on backstepping, for the XY-table. The main contributions of the research presented in this paper are

1. a test for practicability of controllers based on backstepping, emphasis is on the question if transient performance is also improved in the presence of parameter errors and unmodeled dynamics,

2. an extension of the output feedback control scheme to improve its tunability.

1. Corresponding author. Email: A.G.de.Jager@wfw.wtb.tue.nl.
The paper is structured as follows. Section 2 kicks off with a short introduction of tuning functions design. For output feedback two extensions are proposed to obtain more freedom in controller tuning. Section 3 shows the experimental setup, the XY-table, followed by the presentation of the results of state feedback controllers in Section 4. Results of output feedback are in Section 5. Finally, Section 6 winds down the paper with conclusions and directions for future research.

\section{Tuning functions design}

Tuning functions design is an advanced form of adaptive backstepping. For an $n$th order system a controller is recursively designed in $n$ steps. It is assumed that the system model can be written in a parametric strict feedback form, for example

$$
\begin{aligned}
& \dot{x}_{i}=x_{i+1}+\varphi_{i}\left(x_{1}, \ldots, x_{i}\right)^{T} \theta, \quad i=1, \ldots, n-1 \\
& \dot{x}_{n}=b \beta(x) u+\varphi_{n}\left(x_{1}, \ldots, x_{n}\right)^{T} \theta,
\end{aligned}
$$

where $\theta \in R^{p}$ is a vector of unknown constant parameters, $\beta(x)$ and $\varphi_{1}, \ldots, \varphi_{n}$ are smooth nonlinear functions taking arguments in $R^{n}$ with $\beta(x) \neq 0, \forall x \in R^{n}$, and $b$ is an unknown virtual control coefficient called the "high frequency gain." In the $i$ th step, the subsystem consisting of the first $i$ equations of (1) can be stabilized by a proper choice of the "virtual control" $x_{i+1}$ and a parameter update law. This is a traditional Lyapunov based design, with the advantage that the Lyapunov function is known. The virtual control is used to compute a stabilizing function which makes it easier to compute the next virtual control. The parameter update law is not implemented but considered as a tuning function, this function is also used in subsequent steps. At the last step the actual control $u$ is at our disposal, then the parameter update is used. Mathematical descriptions of different tuning functions design methods can be found in [3]. In this section only a mathematical description of a modified K-filter, which is used for state estimation for the output feedback controller, is detailed, because it is changed.

The output feedback control scheme is modified to obtain more freedom in controller tuning. The first extension is a change in the $\mathrm{K}$-filters as described in [3, Section 10.1]. The state estimation filters are presented for linear systems in the observer canonical form

$$
\begin{aligned}
\dot{x}_{1} & =x_{2}-a_{n-1} y \\
& \cdots \\
\dot{x}_{\rho-1} & =x_{\rho}-a_{m+1} y \\
\dot{x}_{\rho} & =x_{\rho+1}-a_{m} y+b_{m} u \\
& \cdots \\
\dot{x}_{n-1} & =x_{n}-a_{1} y+b_{1} u \\
\dot{x}_{n} & =-a_{0} y+b_{0} u \\
y & =x_{1}
\end{aligned}
$$

were $a=\left[a_{n-1}, \ldots, a_{0}\right]^{T}$ and $b=\left[b_{m}, \ldots, b_{0}\right]^{T}$ are vectors of unknown constant parameters. Equations (2) can be written as

$$
\begin{aligned}
& \dot{x}=A x+F(y, u) \theta, \quad x \in R^{n} \\
& y=e_{1}^{T} x
\end{aligned}
$$


where the $p=n+m+1$-dimensional parameter vector $\theta$ is defined by

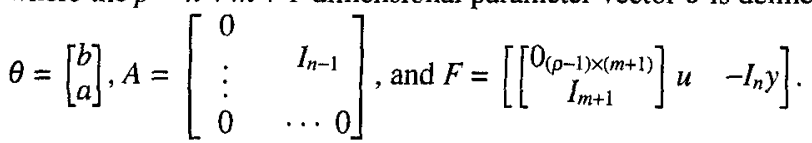

In the absence of full state measurements, filters are needed in the design of an output feedback control law. Two filters are employed for state estimation

$$
\begin{aligned}
\dot{\xi} & =A_{0} \xi+k y \\
\dot{\Omega} & =A_{0} \Omega+F(y, u) C,
\end{aligned}
$$

where $C=\left[\begin{array}{cc}c_{b} & 0_{1 \times(p-1)} \\ 0_{(p-1) \times 1} & I_{(p-1) \times(p-1)}\end{array}\right]$ and the vector $k=\left[k_{1}, \ldots, k_{n}\right]^{T}$ chosen so the matrix $A_{0}=A-k e_{1}^{T}$ is Hurwitz. The vector $e_{i}$ is the $i$ th unit vector in $R^{n}$. A positive constant design parameter $c_{b} \in R$ is added compared to the original scheme. For $c_{b}=1$ this scheme is the same as the original one. Consequences of the extra design parameter are explained later in this section. With these filters the state estimate is

$$
\hat{x}=\xi+\Omega \theta^{*}
$$

where $\theta^{*}=\left[b_{m}^{*}, b_{m-1}, \ldots, b_{0}, a^{T}\right]^{T}$ and $b_{m}^{*}=b_{m} / c_{b}$. Now by substituting (3) and (4) in the derivative of the state estimation error $\varepsilon=x-\hat{x}$, $\dot{\varepsilon}$ can be written as

$$
\dot{\varepsilon}=A_{0} \varepsilon \text {. }
$$

As in [3, Section 10.1], it is possible to lower the dynamic order of the $\Omega$-filter by exploiting the structure of $F(y, u)$. Rewriting the filters as in [3, Section 10.2] results in the filters in Table 1 . The only difference

Table 1: Adapted K-filters

\begin{aligned}$\dot{\eta} & =A_{0} \eta+e_{n} y \quad \eta \in R^{n} \\ \dot{\lambda} & =A_{0} \lambda+e_{n} u \quad \lambda \in R^{n} \\$\hline$\Xi & =-\left[A_{0}^{n-1} \eta, \ldots, A_{0} \eta, \eta\right] \\ v_{j} & =A_{0}^{j} \lambda, j=0, \ldots, m-1 \\ v_{m} & =c_{b} A_{0}^{m} \lambda \\ \Omega & =\left[v_{m}, \ldots, v_{1}, v_{0}, \Xi\right] \\ \xi & =-A_{0}^{n} \eta\end{aligned}$

from the original tuning functions scheme is the parameter $c_{b}$. With these filters the system equations can be prepared for backstepping. The procedure of adaptive backstepping is the same as for the original filters. The only difference is that $b_{m}^{*}$ is estimated instead of $b_{m}$.

The effect of adding the extra parameter $c_{b}$ in the filter design is shown with the help of the equations of the $z$-error system, as computed in $[3$, Section 10.2]. The $z$-error system can be written as

$$
\dot{z}=A_{z}(z, t) z+W_{\varepsilon} \varepsilon_{2}+W_{\theta}(z, t)^{T} \tilde{\theta}^{*}-b_{m}^{*}\left(\dot{y}_{r}+\bar{\alpha}_{1}\right) \tilde{\varrho} e_{1} .
$$

The structure of $A_{z}$ is important. The influence of $c_{b}$ is shown with a second order system. For this system the matrix $A_{z}(z, t)$ is given by

$$
A_{z}(z, t)=\left[\begin{array}{cc}
-c_{1}-d_{1} & b_{m}^{*} \\
-b_{m}^{*} & -c_{2}-d_{2}\left(\frac{\partial \alpha_{1}}{\partial_{y}}\right)^{2}
\end{array}\right],
$$

where $c_{i}$ and $d_{i}$ are positive, constant design parameters. Suppose $A_{z} z$ is dominating in (5), i.e., parameter estimate errors and state estimate errors are small compared to $z$. Then the dynamic behavior of the controlled system is governed by the eigenvalues of $A_{z}$. In the original tuning functions scheme $\left(c_{b}=1\right)$ the eigenvalues cannot be placed freely, because there is no freedom in changing the off-diagonal elements in $A_{z}(z, t)$. The behavior of the controlled system depends on the value of $b_{m}$. For example, when $b_{m}$ is large it is not possible to obtain eigenvalues of $A_{z}$ with a small absolute value. In the modified version $b_{m}^{*}$ appears in the off-diagonal elements. As $b_{m}^{*}$ can be chosen freely, the eigenvalues of $A_{z}$ can be placed freely.

Another extension to the tuning functions design achieves the same effect in the $z$-error system, but results in different dynamic behavior of the controlled system. Simply by applying a change of coordinates to the dynamic system (2) another possibility is achieved in influencing the off-diagonal elements in $A_{z}(z, t)$, as explained below. All the states are scaled by a constant positive design parameter $c_{s}$

$$
\chi_{i}=c_{s} x_{i}, \quad i=1, \ldots, n .
$$

Now the system (3) can be written as

$$
\begin{aligned}
\dot{\chi} & =A \chi+F\left(y^{*}, u\right) \theta^{* *}, \quad \chi \in R^{n} \\
y^{*} & =e_{1}^{T} \chi .
\end{aligned}
$$

where the parameter vector $\theta^{* *}$ is defined by $\theta^{* *}=\left[\begin{array}{c}c_{s} b \\ a\end{array}\right]$.

Applying the backstepping procedure to (6), as described in [3, Chapter 10], results in the same controller with the difference that $c_{s} b$ is estimated instead of $b$ (and $\hat{\varrho}$ becomes the estimate of $1 /\left(c_{s} b_{m}\right)$ ). Now, $c_{s} b_{m}$ appears in the matrix $A_{z}$ instead of $b_{m}$. Again the goal of having complete freedom in placing the eigenvalues of $A_{z}$ is achieved.

This extension can be applied easily to linear systems as the output $y$ appears linearly in the system equations. For nonlinear systems the output $y$ appears in a nonlinear function multiplied with an unknown constant system parameter. Now scaling the states changes the nonlinear function by a factor not the same as the scaling factor. So, then all the parameters $a_{i}$ have to be adjusted.

The closed-loop dynamics with the second modification differs from that of the first modification, in which the filter is adjusted. The reason is that with the second modification, besides the changes in $b$, the magnitude of the error variables $z_{i}$ and the $\lambda$-filter are influenced directly by $c_{s}$. With the first modification, besides $b_{m}$, only the $\lambda$-filter is influenced directly by $c_{b}$. The usefulness of the two extensions will become clear from the experiments with the output feedback controller.

\section{Experimental setup}

A schematic representation of the XY-table is shown in Fig. 1.

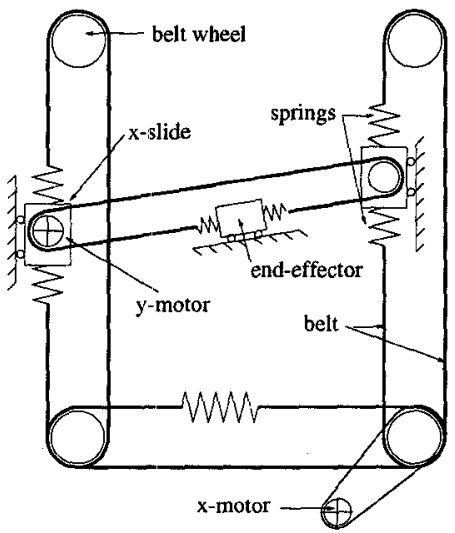

Figure 1: Sketch of the XY-table

The XY-table is an experimental system with several degrees-offreedom, moving in the horizontal plane. Two of the degrees-offreedom are coupled by a spindle with a stiffness that can be varied. This spindle is assumed completely stiff in the model used to design the controllers, as are the springs connecting the belts with the slides. We use a model with two degrees-of-freedom and Coulomb friction, written in strict feedback form, to which backstepping can be applied

$$
\begin{aligned}
& x \text {-direction: } \begin{cases}\dot{x}_{1} & =x_{2} \\
\dot{x}_{2} & =b_{1} u_{1}+\theta_{1} \operatorname{sign}\left(x_{2}\right),\end{cases} \\
& y \text {-direction: } \begin{cases}\dot{y}_{1} & =y_{2} \\
\dot{y}_{2} & =b_{2} u_{2}+\theta_{2} \operatorname{sign}\left(y_{2}\right) .\end{cases}
\end{aligned}
$$


The system parameters of the XY-table are approximately

$$
\begin{array}{ll}
\theta_{1}=1.2[\mathrm{~N} / \mathrm{kg}] & b_{1}=1 / 34[1 / \mathrm{kg}] \\
\theta_{2}=4.4[\mathrm{~N} / \mathrm{kg}] & b_{2}=1 / 2.3[1 / \mathrm{kg}] .
\end{array}
$$

Remark that all rotary movements of the motors, etc., are lumped to the planar movement of the slides and the coordinates $x$ and $y$ correspond with the position of a "target" point on the end-effector. Because the manipulator moves in the horizontal plane there are no gravity forces. The model used for the output feedback controller design is different because in the output feedback control scheme nonlinearities may only depend on the output variable. For that reason, then the Coulomb friction is not modeled and the friction terms in (7) and (8) are omitted.

For satisfactory control of the XY-table several problems have to be taken care of.

1. Motors and amplifiers have their own dynamics and range.

2. The transmission between actuator and slide consists of belts and wheels, which are assumed stiff.

3. Unmodeled viscous friction is present.

4. In the controller design the system parameters are assumed to be constant. This is not true in practice, e.g., the magnitude of the friction depends on the position of a slide on the slide-way.

5. The position measurement of the end-effector is not synchronized with the controller due to limitations in the optical measurement system hardware. This results in an unknown, time dependent, time delay (up to the sample time) of the position measurements.

6. Time delay caused by the computation of the inputs. This is remedied by a Kalman filter which predicts the states one step ahead

7. The differential equations for the dynamic controllers must be solved within the sample interval.

The desired trajectory, used in all experiments, is a circle, $x_{r}=$ $-R \cos (\omega t), y_{r}=R \sin (\omega t)$, were $\omega=\pi t /\left(2 T_{c}^{2}\right)$ for $0 \leq t<2 T_{c}$, $\omega=2 \pi / T_{c}$ for $2 T_{c} \leq t<t_{e n d}$, and $R=0.25[\mathrm{~m}], T_{c}=2$ [s]. During the first cycle the angular frequency increases linearly, starting from zero and smoothly connecting position, velocity, and acceleration, except for $\ddot{y}_{r}$, to the second cycle. Now, too large inputs (larger than the maximum inputs), as a response on initial errors, are avoided. The radius $R$ and the cycle time $T_{c}$ are chosen so the inputs stay in the range were the dynamics of the actuators are approximately linear. For experiments with the $\mathrm{XY}$-table $\mathrm{C}++$ is the programming environment. The controllers are implemented with TCE (Tools for Control Experiments) [6], a collection of small modules in $\mathrm{C}++$ which implement basic tools for control experiments. To compare experiments the Root Mean Square value ( $R M S$ value) of the tracking error is used.

\section{State feedback controller results}

Emphasis in the experiments is on the main goals of research as mentioned in the introduction, verification of practicability and checking the modifications. Experiments were done for two situations

1. control of motor rotations,

2. control of end-effector position.

In most experiments the parameter estimates are initialized at 0 .

\subsection{Control of motor rotations}

The first experiment aims at controlling the motor rotations. For the experiment a torsion spring with a small spring constant of $0.8[\mathrm{Nm} / \mathrm{rad}]$ is used. Tuning of the controller is done by first keeping parameters $c_{i}$ small and choosing the adaptation gains so the estimates of the parameters converge fast to values approximating the real values, without becoming too fluctuating. Then, the transient performance is systematically improved by increasing the parameters $c_{i}$ as far as possible. This tuning resulted in the parameters in Table 2, exp. 1

For the first experiment results are presented in Fig. 2 during the first four cycles, including the cycle with increasing angular frequency. The computed $R M S$ values are $R M S_{x}=0.042$ [mm] and $R M S_{y}$. 0.23 [mm]. The RMS values are computed over the last three cycles. Graphs containing tracking errors and the RMS values show good
Table 2: State feedback controller parameters

\begin{tabular}{cc|ccccc}
\hline Exp. & Dir. & $c_{1}$ & $c_{2}$ & $\kappa$ & $\Gamma$ & $\gamma$ \\
\hline \multirow{2}{*}{1} & $x$ & 100 & 100 & 5 & 100 & 500 \\
& $y$ & 70 & 70 & 5 & 500 & 5 \\
\hline \multirow{2}{*}{2} & $x$ & 5 & 5 & 5 & 100 & 500 \\
& $y$ & 80 & 80 & 5 & 200 & 2 \\
\hline
\end{tabular}

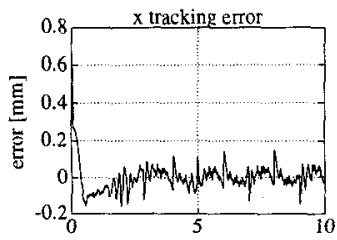

$\mathrm{t}[\mathrm{s}]$

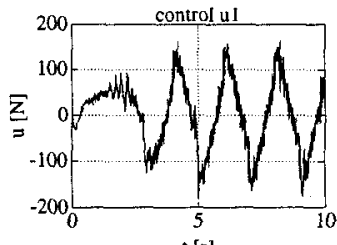

t [s]

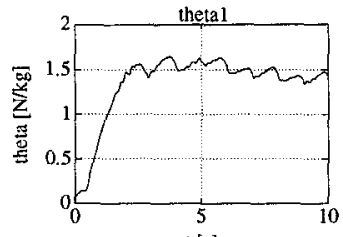

$\mathrm{t}[\mathrm{s}]$

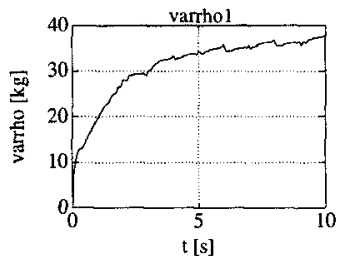

$t[s]$

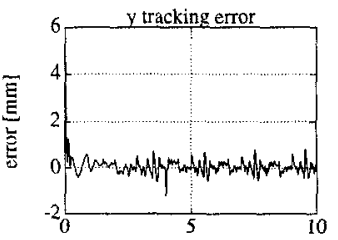

$\mathbf{t}[\mathrm{s}]$

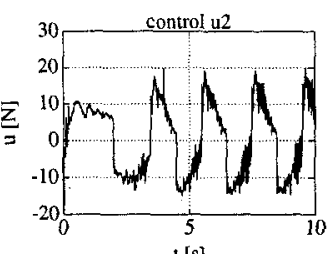

$t[s]$

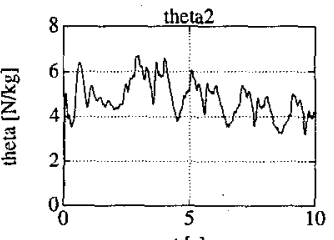

$\mathrm{t}[\mathrm{s}]$

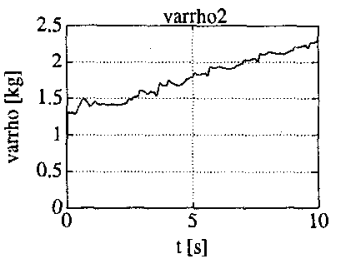

Figure 2: Results, control of motor rotations

transient performance. The tracking errors in $y$-direction are larger than those in $x$-direction. The main cause is the measurement accuracy. Uncertainty in the friction also contributes to the tracking error. For the $y$-direction the friction forces are large relative to the inputs.

The tracking error behavior shows for both directions, especially the $x$-direction, peaks when the velocity changes sign. This is due to the unmodeled dynamics, all unmodeled springs and belts first have to be tightened before movement of the slide is possible again. During the first cycle the parameter estimates, especially in $y$-direction, change fast. After that, the parameter estimates converge slowly.

The computed inputs are a bit fluctuating, this is due to the controller's response on errors in the state prediction by the Kalman filter At every sample point errors are present in the predicted states, mainly due to measurement errors and unmodeled dynamics (for example backlash).This is also the reason why the transient performance cannot be improved indefinitely. Increasing the parameters $c_{i}$ will cause a better tracking error performance (theoretically), but will also cause the state estimation errors to become more important. First, these errors become relative large and, secondly, the controller reacts stronger on them. This results in a strongly fluctuating input. If the fluctuations become too large the actuator saturates, resulting in worse transient performance. 


\subsection{Control of end-effector position}

The experiments in the previous section did aim at tracking a reference for the motor rotations. This is, especially when the torsion spring is really flexible, not the same as control of the position of the end-effector. In this section attempts are made to control the position of the end-effector directly. Besides the extra influence of unmodeled dynamics, another problem is the end-effector position. This is acquired by a dedicated computer which needs time to process the raw data of a CCD camera, approximately the same as the sampling time $T_{s}=0.002$ [s]. This introduces an unknown and changing time delay. Because of the extra flexibility (introducing more degrees-of-freedom) and larger measurement errors the closed-loop system is more likely to become unstable, therefore the control parameters are adjusted so the controller becomes less aggressive. This is achieved by decreasing $c_{1}$ and $c_{2}$ for the $x$-direction and decreasing the adaptation gains in $y$-direction, giving the design parameters in Table 2, exp. 2 .

Again a torsion spring with a small spring constant of $0.8[\mathrm{Nm} / \mathrm{rad}]$ is used. Results of the experiment are presented in Fig. 3. The graphs

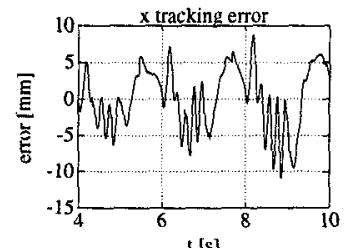

! $[\mathrm{s}]$

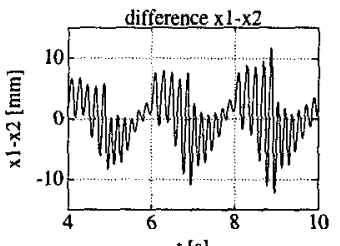

$\mathrm{t}$ [s]
Figure 3: Results, control of end-effector position

show the tracking error in $x$-direction and the difference between the two $x$-slides $x_{1}-x_{2}$. The latter shows the flexibility, $x_{1}$ and $x_{2}$ differ up to $10[\mathrm{~mm}]$. The tracking error behavior in $y$-direction was similar to the results of controlling the motor rotations. Tracking errors were slightly larger due to the measurement equipment and to the increased influence of the unmodeled dynamics. Computed $R M S$ values of the tracking errors are $R M S_{x}=4.2[\mathrm{~mm}]$ and $R M S_{y}=0.39[\mathrm{~mm}]$.

The torsion spring imposes a limit on the performance. The influence of the torsion spring is clearly shown in the graph containing the difference $x_{1}-x_{2}$. The closed-loop system is most likely to become unstable when the end-effector is near the $x_{2}$ slide, which is not directly driven. Increasing any one of the control parameters will result in a stronger fluctuating difference $x_{1}-x_{2}$. Increasing the parameters is possible till the fluctuations become that large that the belts start skipping teethes on the belt-wheel.

Tuning of the parameters influences the dynamics of the closedloop system. So it could be expected that proper tuning results in a better behavior of the controlled system. Several ways of improving the transient performance have been tried for the XY-table (e.g., starting with all controller parameters small and increasing one or few of them as far as possible). None of the tunings led to the desired tracking error behavior with bounded and not too large difference $x_{1}-x_{2}$ and small tracking errors. The use of a three degrees-of-freedom model for the controller design might be a good solution for satisfactory control of the end-effector position of the flexible XY-table. However, two difficulties have to be overcome, (1) there is no general extension of the theory for the control of MIMO systems yet, and (2) the system equations must be written in a parametric pure feedback form.

As mentioned, the torsion spring has a small spring constant. Experiments show that when increasing the spring constant, at some point the springs connecting the belts to the slides impose a limit on the controller tuning and performance, instead of the torsion spring.

\section{Output feedback controller results}

Before describing experiments for the XY-table the influence of the new design parameters is discussed.

Limited freedom in the design of the output feedback controller is already established in Section 2. Simulations have proven the expectations. If the initial errors are large, the term with $A_{z}$ dominates in (5).
In $A_{z}$ the off-diagonal elements are the high frequency gains $b_{i}$, which are large for the simulated XY-table. If the controller is chosen fast (i.e., large $d_{i}$ and $c_{i}$ ) the closed-loop response is aggressive, resulting in large control efforts, too large to be of practical use. It would be desirable if by decreasing any one (or a combination) of the controller parameters the response could be shaped at will. However, this does not happen. As can be expected from (5) the tracking error becomes more fluctuating if controller parameter(s) are decreased, also resulting in large inputs. So, with the original controller no satisfying control of the XY-table can be obtained in the presence of large initial errors. By influencing the off-diagonal elements in $A_{z}$ by use of one of the two new tunable parameters, it was possible to obtain the desired shape of the response without large control efforts. This is a new and desirable feature of the controller.

Increasing any one of the parameters $c_{i}$ and $d_{i}$ improves the transient performance, but the absolute values of the eigenvalues of the (linearized) differential equations increase rapidly, making these equations more difficult to solve accurately in real time. Now, by using both extensions proposed in Section 2, it is possible to place the eigenvalues of the differential equations and $b_{i}^{*}=b_{i} c_{s} / c_{b}$ freely. In a restricted parameter range the differential equations are easy to solve by using simple methods. In all experiments first order Euler integration is used.

Again, experiments with the XY-table were performed for the two same cases. The same desired trajectory as in the previous section, with in the first cycle an increasing angular frequency, is used.

\subsection{Control of motor rotations}

In previous experiments the position was measured, from this measurement the position and velocity were predicted one step ahead by a Kalman filter. Now, the same filter is used to predict only the position one step ahead, and not the velocity. In the previous section, the errors remaining after filtering also imposed a limit on performance. If the filter states, especially the velocity prediction, are not used, performance may be improved. As the output feedback controller only uses the position prediction, it might perform better.

Tuning the controller is done by first giving $c_{b}$ and $c_{s}$ values so the differential equations are easy to solve and $b_{i}^{*}$ does not become too large. The adaptation gains were chosen not too large to prevent fast rising estimates. By increasing $c_{i}$ and $d_{i}$ the tracking error performance was improved, resulting in the parameters in Table 3, exp. 1.

Table 3: Output feedback controller parameters

\begin{tabular}{cc|cccccc}
\hline Exp. & Dir. & $c_{1}=c_{2}$ & $d_{1}=d_{2}$ & $c_{b}$ & $c_{s}$ & $n$ & $\gamma_{2}$ \\
\hline \hline \multirow{2}{*}{1} & $x$ & 200 & 100 & $1.10^{-6}$ & $1.10^{-4}$ & $1.10^{8}$ & $1.10^{5}$ \\
& $y$ & 200 & 30 & $5.10^{-5}$ & $5.10^{-4}$ & $1.10^{7}$ & $1.10^{5}$ \\
\hline \multirow{2}{*}{2} & $x$ & 100 & 50 & $1.10^{-6}$ & $1.10^{-4}$ & $1.10^{8}$ & $1.10^{5}$ \\
& $y$ & 50 & 25 & $5.10^{-5}$ & $5.10^{-4}$ & $1.10^{7}$ & $1.10^{5}$ \\
\hline
\end{tabular}

Initial parameter estimates are chosen as $\hat{\varrho}_{i}(0)=1 / \hat{b}_{i}^{*}(0)=1$. By setting both $k_{1}$ and $k_{2}$ equal to 2 , the matrix $A_{0}$ is Hurwitz. The results of the controller applied to the XY-table are presented in Fig. 4 during the first four cycles. Computed $R M S$ values of the tracking error are $R M S_{x}=0.54[\mathrm{~mm}]$ and $R M S_{y}=3.5[\mathrm{~mm}]$.

Compared to the state feedback controller the tracking errors are large. Achieving better transient performance seems not possible in the restricted parameter range. Any further increase of the parameters $c_{i}$, $d_{i}$, will cause the differential equations to become too stiff. The input in $y$-direction already shows consequences of the limited accuracy of solving the differential equations. Increasing $c_{i}$ and/or $d_{i}$ would make the variations in the input signal worse.

\subsection{Control of end-effector position}

For this controller too, an attempt is made to control the position of the end-effector. Because this closed-loop is sensitive for instabilities, the parameters $c_{i}$ and $d_{i}$ are decreased. Settings are summarized in Table 3, exp. 2. The experiment is for the rigid XY-table (torsion spring is replaced by a rigid shaft), so the results, presented in Fig. 5 for three 

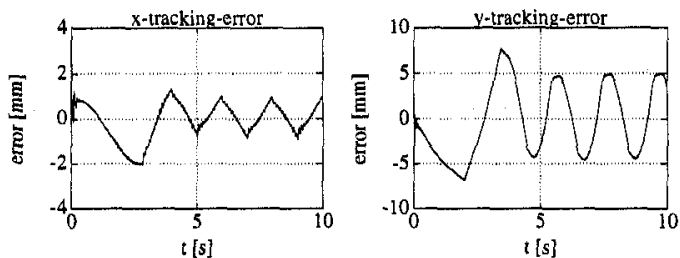

control ul
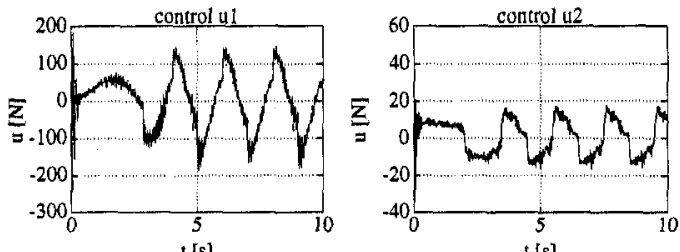

$\mathfrak{t}[\mathrm{s}]$
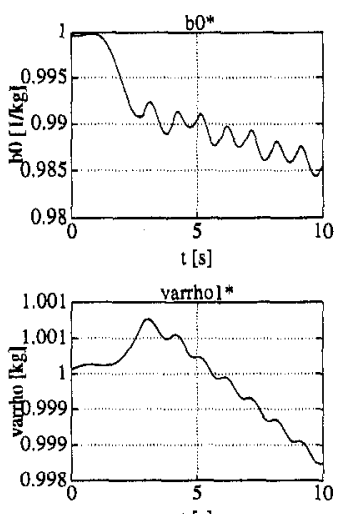

$\mathrm{t}[\mathrm{s}]$

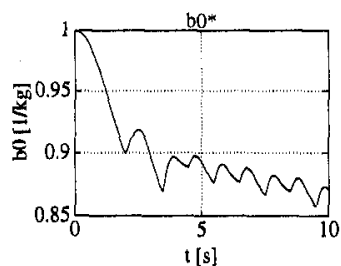

t

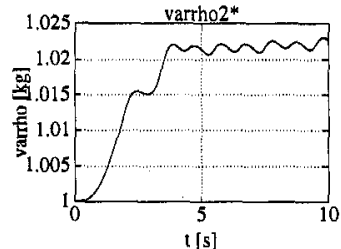

t $[\mathrm{s}]$

Figure 4: Results, control of motor rotations

cycles, are not comparable with the previous ones. The computed $R M S$ values are $R M S_{x}=3.6[\mathrm{~mm}]$ and $R M S_{y}=4.9[\mathrm{~mm}]$.
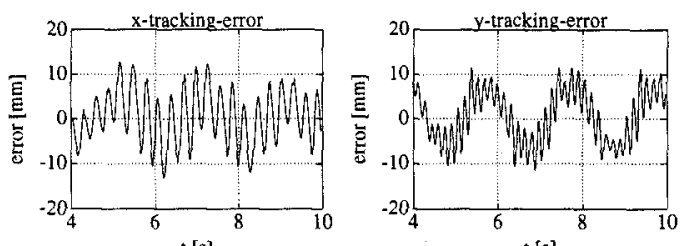

$\mathrm{t}[\mathrm{s}]$

$t$ s $]$

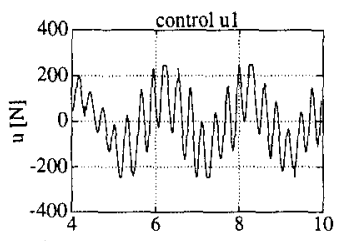

$\mathrm{t}[\mathrm{s}]$

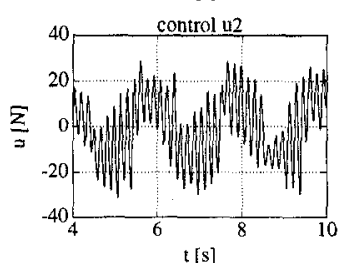

Figure 5: Results, control of end-effector position

A feature of the results is that the tracking error and input, for both directions, are fluctuating. The computed input $u_{1}$ exceeds the maximum input a few times (e.g., at $t=5.2[\mathrm{~s}]$ and $6.2[\mathrm{~s}]$ ). This did not happen in the previous experiments.

Further investigation showed that the fluctuations in $y$-direction were mainly caused by the shifting time delay, due to the optical measurement hardware. Checking the influence of the different sources of unmodeled dynamics showed that in $x$-direction mainly the unmodeled Coulomb friction caused the fluctuations.

If the unmodeled dynamics are reduced (sampling and measuring are synchronized and the friction is partly compensated) there is improvement, but the controller parameters still cannot be chosen so the tracking error becomes acceptable. Obviously, the controller cannot handle the remaining unmodeled dynamics. If the controller parameters are adjusted, so the transient performance theoretically improves, the controller will induce a more fluctuating tracking error behavior and larger control inputs. The controller can be made more aggressive until the belt starts skipping teethes on the belt wheel or the maximum inputs are exceeded, but even then the error is not good enough.

The freedom in the design of an output feedback controller is limited to the range where the differential equations are easy to solve. Therefore, the conclusions of the output feedback controller are also limited. In simulations (not presented) the controller seemed well capable of handling the unmodeled Coulomb friction. However, in simula. tions there was more freedom in the design of the controller, real time computation not being an issue, the Coulomb friction was constant and, besides that, the only unmodeled part was the torsion spring.

\section{Conclusions and recommendations}

Here the most important conclusions are summarized and recommendations for further research are given.

- Experiments indicated good practicability of the state feedback controller.

- The output feedback controller could be implemented in a restricted controller parameter range only, due to the stiffness of the differential equations. In that range the results were inferior to the results obtained by the state feedback controller.

- The extensions proposed in this report proved to be successful, more freedom in the design of the controller was obtained, and it provided also a tool to influence the stiffness of the differential equations.

- In the experiments for control of the end-effector position, the closed-loop system could become unstable, due to the springs connecting the belts to the slides or due to the torsion spring. For the rigid XY-table and using state feedback, control of the end-effector position was improved by controlling the measured end-effector position. For the flexible XY-table, best tracking error behavior of the end-effector, in $x$-direction, was achieved if the motor rotations were controlled.

- Control of the end-effector position seems open for improvement. The following modifications seem promising, (1) decrease the time delay due to the measurement hardware, (2) compute the endeffector position with the measurements of the positions of the 3 slides, or (3) use a 3 degrees-of-freedom model in controller design.

- Especially the results with the state feedback controller encourage further research in controllers based on tuning functions design. More advantages of these controllers may appear if the controllers are implemented on a strongly nonlinear system.

\section{References}

[1] B. de Jager and J. Banens, "Experimental evaluation of robot controllers," in Proc. of the 33rd IEEE Conf. on Decision and Control, vol. 1, (Lake Buena Vista, FL), pp. 363-368, IEEE, Piscataway, NJ, Dec. 1994

[2] I. Kanellakopoulos, "Passive adaptive control of nonlinar systems," Internat. J. of Adaptive Control and Signal Processing, vol. 7, pp. 339$352,1993$.

[3] M. Krstić, I. Kanellakopoulos, and P. Kokotović, Nonlinear and Adaptive Control Design. John Wiley \& Sons, New York, 1995.

[4] M. Krstić, "Adaptive nonlinear control," 1994. PhD Dissertation, University of California, Santa Barbara.

[5] M. Krstić, I. Kanellakopoulos, and P. V. Kokotović, "Adaptive control without overparametrization," System and Control Letters, vol. 19, pp. 177-185, 1992.

[6] J. Banens and B. de Jager, "TCE: a software environment for real-time control," in Preprints of the 3rd IFAC/IFIP workshop on Algorithms and Architectures for Real-Time Control (AARTC'95) (P. Fleming and L. Boullart, eds.), (Ostend, Belgium), pp. 303-308, BIRA, 1995. 